\title{
SPAG5: an emerging oncogene
}

Ji He, ${ }^{1}$ Andrew R. Green, ${ }^{2}$ Yan Li, ${ }^{1}$ Stephen Y. T. Chan, ${ }^{3}$ and Dong-Xu Liu ${ }^{1, *}$

${ }^{1}$ The Centre for Biomedical and Chemical Sciences, School of Science, Faculty of Health and Environmental Sciences, Auckland University of Technology, Auckland, New Zealand

${ }^{2}$ Nottingham Breast Cancer Research Centre, Division of Cancer and Stem Cells, School of Medicine, University of Nottingham Biodiscovery Institute, Nottingham, UK

${ }^{3}$ Department of Clinical Oncology, University of Nottingham and Nottingham University Hospitals NHS Trust, Nottingham, UK

*Correspondence: dong-xu.liu@aut.ac.nz (D. X. Liu).

\section{Keywords}

SPAG5, astrin, oncogene, gene amplification, therapeutic target, cancer therapy

\begin{abstract}
SPAG5 is a mitotic spindle protein. Recent studies have found that it is overexpressed in many human cancers and functions as an oncogene. We summarize the current underlying mechanisms for its oncogenic roles in regulating cellular behaviors of cancer cells and discuss the possibility to target SPAG5 for cancer treatment.
\end{abstract}

\section{SPAG5 in human cancers}

Sperm associated antigen 5 (SPAG5, also called astrin) is a mitotic spindle protein. In normal cells, it ensures a correct separation of sister chromatids into daughter cells. SPAG5 depletion induces a spindle checkpoint arrest characteristic of the formation of multipolar spindles and a loss of sister chromatid cohesion [1]. However, SPAG5 gene gains and amplifications are observed in many cancers (Table 1). Increased SPAG5 expression is correlated with poor clinical outcomes and adverse clinicopathological features in breast cancer [2]. In several cancer models, SPAG5 is implicated in neoplastic growth, chemoresistance, metastasis, local recurrence and overall survival, whereas its downregulation results in suppressed cell 
proliferation, impaired migration motility and enhanced apoptosis and chemosensitivity [2-7]. In addition, SPAG5 can be used as an antigen for cancer vaccine development [8]. These observations clearly indicate that SPAG5 is a novel driver oncogene and may be a therapeutic target. Herein, we summarize the current signaling pathways that are regulated by SPAG5 and those that mediate the cellular functions of SPAG5 in cancer cells and propose a strategy to target SPAG5 for cancer therapy.

\section{SPAG5 Signaling in Cancer}

Compelling evidence has demonstrated that SPAG5 is involved in various intracellular processes that regulate tumor initiation and progression, in addition to counteracting the cytotoxic effects of many chemotherapies.

Oxidative stress is a prevalent condition in tumors. The mammalian target of rapamycin (mTOR) complex 1 (mTORC1) is activated in response to stress and promotes the biosynthesis of stress response proteins. Upon metabolic challenge and oxidative stress, the deprivation of tuberous sclerosis proteins 1 and 2 (TSC1-TSC2) activates mTORC1 and upregulates SPAG5 levels. In addition to mitosis-related functions, SPAG5 inhibits mTOR-raptor association and recruits raptor to stress granules (SGs), inhibiting mTORC1 activity (Figure 1A). Thus, TSC1TSC2 combined with SPAG5 keep the cellular level of mTORC1 under control. This balanced mTORC1 activity enables expression of stress response proteins that are required for cell survival under stress, and prevents cells from undergoing mTORC1 hyperactivation-induced apoptosis [9]. However, hyperactivation of mTORC1 induced by lack of functional SPAG5 may increase hypoxia tolerance by promoting the biosynthesis of hypoxia-inducible factor $1 \alpha$ (HIF-1 $\alpha$ ) in cancer cells [10], and autophagy by phosphorylating autophagy regulatory complex containing unc-51-like kinase 1 (ULK1), the mammalian ATG13 protein and focal adhesion kinase interacting protein of $200 \mathrm{kDa}$ (FIP200) [11]. Further studies are required to investigate to what extent SPAG5-mediated hyperactivation of mTORC1 influences autophagy and hypoxia tolerance in cancer cells in the absence of functional SPAG5.

SPAG5 is also found to prohibit apoptosis through the p53-mediated DNA damage response pathway, which modulates cellular responses to DNA damage, redox stress, or abnormal oncogene amplification, and promotes several pro-apoptotic genes, such as, BAX, PUMA, and NOXA (Figure 1A). Upon genotoxic stresses, upregulated p53 induces BAX expression and elevated caspase-3 activity in the absence of functional SPAG5 [12]. The upregulation of p53 also inhibits mTORC1 activity via the TSC1-TSC2 complex, leading to 
interference in protein synthesis [12]. Thus, in tumor cells overexpression of SPAG5 coupled with suppression of p53 result in an environment favoring cell growth and proliferation when challenged with DNA lesions.

Secreted Wnt3 protein plays an oncogenic role in cancers. SPAG5 upregulates Wnt3 by activating the AKT/mTOR signaling (Figure 1B) [7]. Accordingly, cancer cells overexpressing SPAG5 that are adequate in Wnt3 expression display elevated levels of cell proliferation, invasion and chemoresistance even after exposure to agents that induce cell cycle arrest [13]. This pro-proliferative effect is due to the dephosphorylation of $\beta$-catenin induced by ligand binding of Wnt3. $\beta$-catenin is able to translocate into the nucleus in its dephosphorylated state and form a complex with T cell factor (TCF), which can activate gene transcription, including of oncogenes. This canonical Wnt $3 / \beta$-catenin signaling pathway can transactivate the expression of epidermal growth factor receptor (EGFR), which is related to chemoresistance to trastuzumab in human epidermal growth factor receptor 2 (HER2) overexpressing breast cancer cells [13]. It also confers resistance to the chemodrug cisplatin in bladder cancer [7]. Furthermore, the translocation of $\beta$-catenin plays a significant role in regulating epithelial-mesenchymal transition [13]. The transition could promote not only tumor invasion and metastases, but also resistance to tamoxifen in estrogen receptor positive breast cancer cell lines.

Centrosome protein 55 (Cep55) helps physical separation of dividing cells in the final stage of the cell cycle, a process called cytokinesis. Recent work demonstrated that Cep55 facilitates cell proliferation and migration through activation of PI3K/AKT/mTOR pathway. SPAG5 enhances this promoting effect by its binding to Cep55 (Figure 1C). In normal healthy cells, balanced expression of SPAG5 mediates Cep55, making cell division and proliferation controllable. In tumor cells, however, the overexpression of SPAG5 makes this protein-protein interaction detrimental since it stimulates activation of many downstream effectors that favor cell proliferation and survival [14].

The scavenger receptor class A member 5 (SCARA5) is a tumor suppressor gene that suppresses progression and metastasis of cancer. Converging evidence shows that SCARA5 expression is frequently downregulated in cancer cells, and suppression of SPAG5 leads to a rise in SCARA5 levels, which in turn, slows down cell progression and promotes apoptosis (Figure 1D). It is proposed that SPAG5 downregulates SCARA5 expression via activation of the $\beta$-catenin/TCF4 pathway. As aforementioned, SPAG5 can typically result in dephosphorylation and translocation of $\beta$-catenin through the AKT/mTOR/Wnt axis. In combination with TCF4, degraded $\beta$-catenin further mediates gene transcription, thereby 
downregulating SCARA5 expression. Thus, the proliferation of cancer cells with aberrant SPAG5 expression is accelerated due to the role played by SPAG5 in SCARA5 downregulation $[6]$.

Emerging evidence demonstrates that SPAG5 may confer resistance to olaparib in triple-negative breast cancer (Figure 1E) [3]. c-MYC is a crucial oncogenic transcription factor mediating gene transcription that is involved in multiple cellular mechanisms, such as cell proliferation, cell apoptosis, cell-cycle control, and DNA repair. SPAG5 can enhance the transcriptional activity of c-MYC through increasing and interacting with c-MYC binding protein (MYCBP). c-MYC could then directly upregulate the expression of homologous recombination (HR) DNA repair proteins RAD51, BRCA1 and BRCA2, which confer resistance to olaparib by repairing the DNA lesions due to inhibited poly-ADP ribose polymerase (PARP) activity. Another mechanism through which SPAG5 confers resistance to olaparib is through mediating the cell cycle. SPAG5 promotes S/G2 transition, leading to a reduced S-phase duration in which olaparib-provoked DNA lesions occur. Therefore, SPAG5 may be used as a potential biomarker to stratify patients for olaparib treatment [3].

In summary, SPAG5 modulates cell proliferation and migration/metastasis through the activation of the PI3K/AKT/mTOR pathway and its downstream effectors such as Wnt signaling. SPAG5 also affects cell growth and apoptosis by downregulating p53 and mTORC1 signaling and upregulating c-MYC signaling. As a result of decreased cell death and increased proliferation, cancer cells become more resistant to chemodrugs.

\section{Upstream Regulators of SPAG5}

SPAG5 is regulated by many upstream regulators, including glycogen synthase kinase $3 \beta$ (GSK3 $\beta$ ), human Ninein (hNinein), Aurora B kinase, OSBP-related protein 8 (ORP8), p29, sensitive to nitrogen mustard 1A (SNM1A), and Mid2 ubiquitin ligase. A few studies have reported the oncogenic functions of SPAG5 are mediated by microRNAs (miRNAs) (Figure 1F). miR-539 directly binds the 3' untranslated region of SPAG5 mRNAs and downregulates protein expression, resulting in the inhibition of prostate cancer cell proliferation, invasion, migration, as well as tumor growth and metastasis [5]. Similarly, miR-363-3p targets SPAG5 and modulates its expression in hepatocellular cancer cell, affecting cell proliferation and migration via the SPAG5/CEP55/PIK3k/AKT axis [14]. In non-small cell lung cancer, miR1179 also regulates SPAG5 expression and suppresses cell growth and invasion by targeting SPAG5/AKT signaling [15]. These studies indicate that miRNAs play a critical role in controlling SPAG5 expression. 


\section{Concluding Remarks and Future Perspectives}

It has become clear that SPAG5 utilizes several mechanisms to exert its oncogenic effects. Given the essential role of SPAG5 in mitosis, SPAG5 antagonism may represent a novel strategy for anticancer therapy, especially in reversing chemoresistance. This is very promising considering that SPAG5 expression alters sensitivity to taxol in cervical cancer [4] and SPAG5 knockdown results in enhanced tumor sensitivity to olaparib in triple-negative breast cancer [3]. Olaparib, as a PARP inhibitor for cancer therapy, relies on an exploitation of the defective DNA damage repair mechanism due to mutations and epigenetic changes of HR genes including BRCA1/2. SPAG5 antagonism may improve the efficacy of olaparib because SPAG5 knockdown increased S-phase cell population and decreased the expression of DNA repair proteins RAD51 and BRCA1/2 [3].

One obstacle that prevents the use of SPAG5 as a drug target is its intracellular localization and lack of enzymatic activity, which makes it difficult to use antibodies or catalytical activity inhibitors to neutralize the function of SPAG5. Although antibodies and small molecular inhibitors dominate the approved drugs, there are many novel approaches to making intracellular proteins druggable. They include small peptide inhibitors, siRNAs and miRNAs, which can penetrate the membrane barriers. Moreover, antibodies against intracellular proteins may be internalized into cells by normal and pathological processes, even by direct delivery using various technologies including nanoparticle carriers. Future perspectives thus include the development of anti-SPAG5 therapeutic agents and effective delivery of them into cancer cells.

\section{Acknowledgements}

The work was supported by the Nottingham Hospitals Charity to S.Y.T.C. and D.-X.L.'s research is supported by the Breast Cancer Foundation NZ and the Breast Cancer Cure.

\section{Disclaimer Statement}

S.Y.T.C is named inventor on patent application US20170138947A1. The other authors declare no conflict of interest. 


\section{References}

1. Thein, K.H. et al. (2007) Astrin is required for the maintenance of sister chromatid cohesion and centrosome integrity. J Cell Biol 178, 345-54.

2. Abdel-Fatah, T.M.A. et al. (2016) SPAG5 as a prognostic biomarker and chemotherapy sensitivity predictor in breast cancer: a retrospective, integrated genomic, transcriptomic, and protein analysis. Lancet Oncol 17, 1004-1018.

3. Li, M. et al. (2019) SPAG5 upregulation contributes to enhanced c-MYC transcriptional activity via interaction with c-MYC binding protein in triple-negative breast cancer. J Hematol Oncol 12, 14.

4. Yuan, L.J. et al. (2014) SPAG5 upregulation predicts poor prognosis in cervical cancer patients and alters sensitivity to taxol treatment via the mTOR signaling pathway. Cell Death Dis 5, e1247.

5. Zhang, H. et al. (2016) miR-539 inhibits prostate cancer progression by directly targeting SPAG5. J Exp Clin Cancer Res 35, 60.

6. Liu, H. et al. (2018) SPAG5 promotes hepatocellular carcinoma progression by downregulating SCARA5 through modifying beta-catenin degradation. $J$ Exp Clin Cancer Res 37, 229.

7. Liu, J.Y. et al. (2018) SPAG5 promotes proliferation and suppresses apoptosis in bladder urothelial carcinoma by upregulating Wnt3 via activating the AKT/mTOR pathway and predicts poorer survival. Oncogene 37, 3937-3952.

8. Li, B. et al. (2016) Comprehensive analyses of tumor immunity: implications for cancer immunotherapy. Genome Biol 17, 174.

9. Thedieck, K. et al. (2013) Inhibition of mTORC1 by Astrin and Stress Granules Prevents Apoptosis in Cancer Cells. Cell 154, 859-874.

10. Saxton, R.A. and Sabatini, D.M. (2017) mTOR Signaling in Growth, Metabolism, and Disease. Cell 169, 361-371.

11. Chen, Y. et al. (2014) Hyperactivation of mammalian target of rapamycin complex 1 (mTORC1) promotes breast cancer progression through enhancing glucose starvation-induced autophagy and Akt signaling. J Biol Chem 289, 1164-73.

12. Yang, Y.C. et al. (2006) Silencing of astrin induces the p53-dependent apoptosis by suppression of HPV18 E6 expression and sensitizes cells to paclitaxel treatment in HeLa cells. Biochem Biophys Res Commun 343, 428-34.

13. Wu, Y. et al. (2012) Expression of Wnt3 activates Wnt/ $\beta$-catenin pathway and promotes EMT-like phenotype in trastuzumab-resistant HER2-overexpressing breast cancer cells. Mol Cancer Res 10, 1597-606.

14. Yang, Y.F. et al. (2018) SPAG5 interacts with CEP55 and exerts oncogenic activities via PI3K/AKT pathway in hepatocellular carcinoma. Mol Cancer 17, 117.

15. Song, L. et al. (2018) MicroRNA-1179 suppresses cell growth and invasion by targeting sperm-associated antigen 5-mediated Akt signaling in human non-small cell lung cancer. Biochem Biophys Res Commun 504, 164-170. 
Table 1. SPAG5 copy number gain and amplification in cancer. Data were extracted from the COSMIC cancer database (https://cancer.sanger.ac.uk/cosmic).

\begin{tabular}{|c|c|c|c|c|}
\hline \multirow[t]{2}{*}{ Cancer } & \multicolumn{2}{|c|}{$S P A G 5$ copy number gain } & \multicolumn{2}{|c|}{ SPAG5 overexpression } \\
\hline & Percentage $(\%)$ & Cases (n) & Percentage $(\%)$ & Cases $(n)$ \\
\hline Adrenal gland & & - & 7.59 & 79 \\
\hline Breast & 2.35 & 1492 & 16.76 & 1104 \\
\hline $\begin{array}{l}\text { Central } \\
\text { nervous } \\
\text { system }\end{array}$ & & - & 9.33 & 697 \\
\hline Cervix & & - & 3.58 & 307 \\
\hline Endometrium & 0.34 & 586 & 11.96 & 602 \\
\hline $\begin{array}{l}\text { Hematopoietic } \\
\text { and lymphoid }\end{array}$ & & - & 8.6 & 221 \\
\hline Kidney & & - & 11.17 & 600 \\
\hline Large intestine & 0.42 & 717 & 5.74 & 610 \\
\hline Liver & 0.45 & 663 & 5.9 & 373 \\
\hline Lung & 0.3 & 1006 & 10.89 & 1019 \\
\hline Esophagus & 0.2 & 510 & 10.4 & 125 \\
\hline Ovary & 1.02 & 684 & 2.63 & 266 \\
\hline Pancreas & & - & 7.26 & 179 \\
\hline Prostate & & - & 10.84 & 498 \\
\hline Skin & 0.17 & 587 & 5.92 & 473 \\
\hline Soft tissue & & - & 9.13 & 263 \\
\hline Stomach & 0.64 & 472 & 7.72 & 285 \\
\hline Thymus & 0.81 & 124 & & - \\
\hline Thyroid & & - & 4.29 & 513 \\
\hline $\begin{array}{c}\text { Upper } \\
\text { aerodigestive } \\
\text { tract }\end{array}$ & 0.19 & 520 & 7.85 & 522 \\
\hline Urinary tract & 1.25 & 399 & 16.67 & 408 \\
\hline
\end{tabular}


(A)

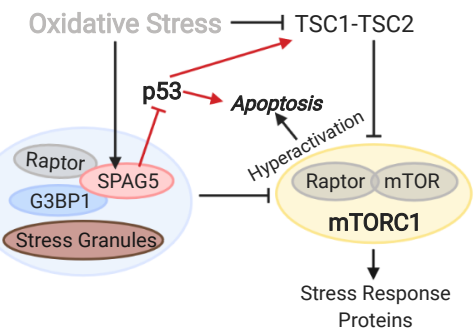

(D)

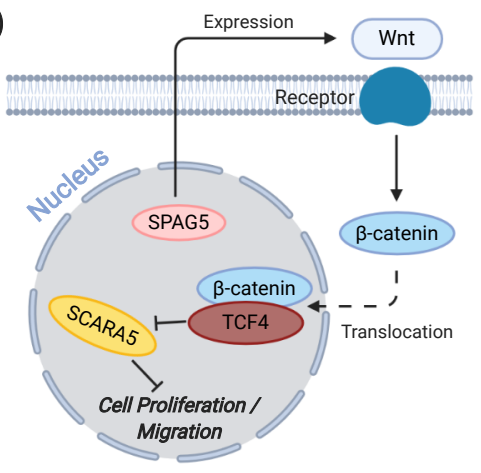

(B)

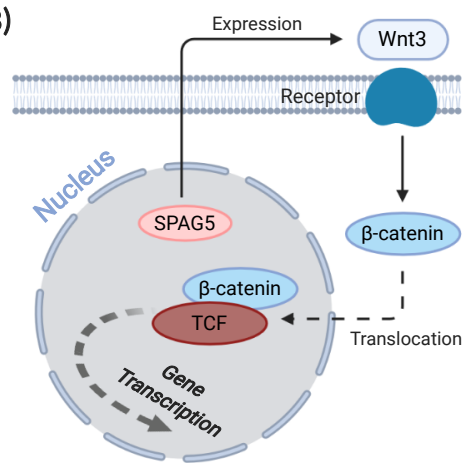

(E)

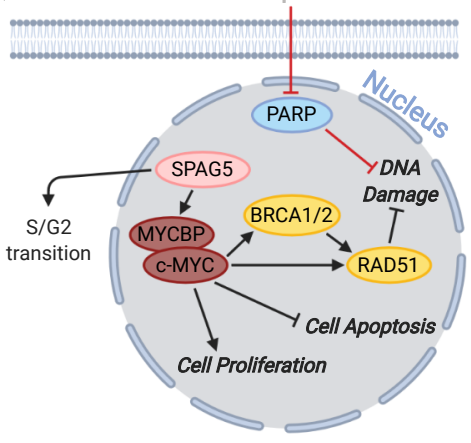

(C)

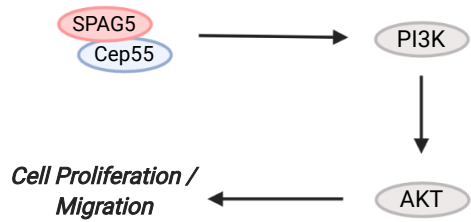

(F)

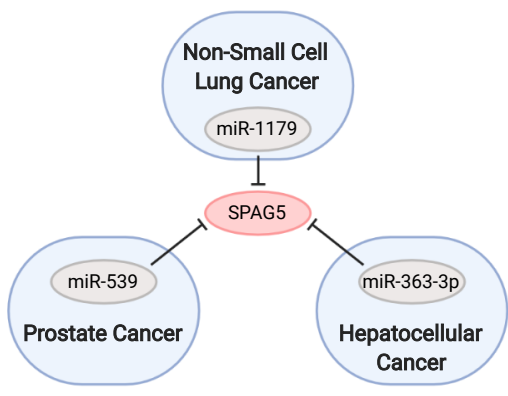


(A)

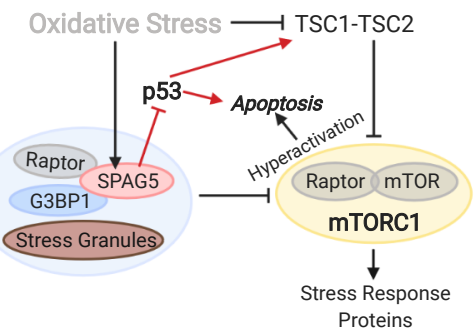

(D)

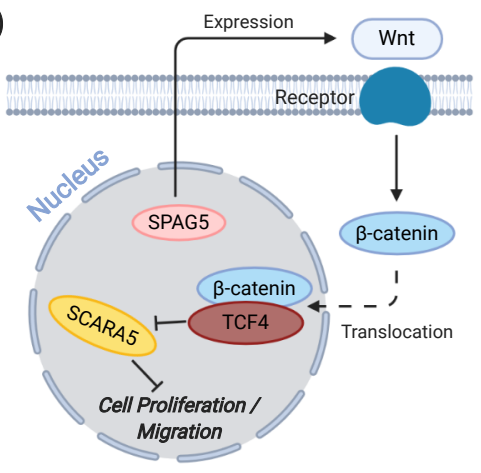

(B)

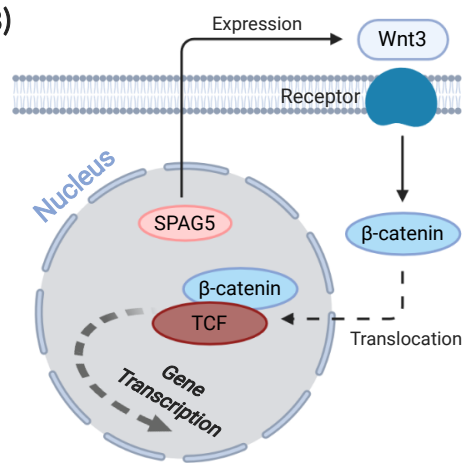

(E)

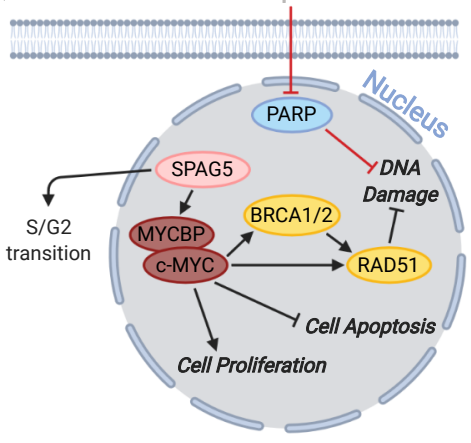

(C)

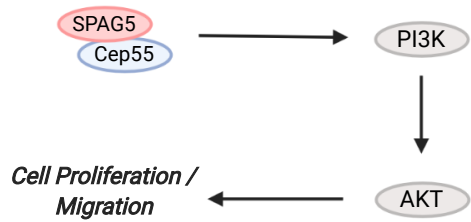

(F)

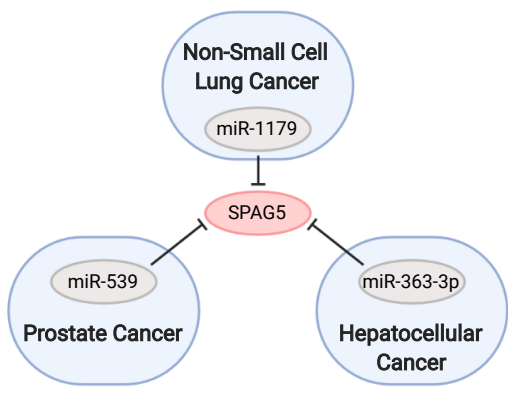


Figure 1. Current understanding of SPAG5 signaling in cancer. (A) Sperm associated antigen 5 (SPAG5) is involved in the regulation of mammalian target of rapamycin complex 1 (mTORC1). Upon oxidative stress, SPAG5 and stress granules (SG) complex coupled with downregulated tuberous sclerosis proteins 1 and 2 (TSC1-TSC2) lead to a balanced activity of mTORC1. Hyperactivation of mTORC1 causes apoptosis whereas a balanced mTORC1 activity promotes cell survival under stress. SPAG5 also prevents cancer cells from undergoing apoptosis by inhibiting p53. (B) SPAG5 is implicated in the expression of Wnt3. $\beta$-catenin, which is mediated by Wnt3, interacts with T cell factor (TCF) and promotes the transcription of oncogenes that regulate progression, metastasis and chemoresistance of cancer cells. (C) Binding of SPAG5 to centrosome protein 55 (Cep55) activates PI3K/AKT/mTOR (phosphatidylinositol 3-kinase/protein kinase B/mammalian target of rapamycin) signaling, which is related to cancer cell progression, metastasis, and chemoresistance. (D) Scavenger receptor class A member 5 (SCARA5) is an anti-oncogene that suppresses progression and metastasis of cancer. Upregulation of SPAG5 may thus promote the proliferation and metastasis of cancer cells by inhibiting SCARA5 through Wnt/ $\beta$-catenin/TCF4 axis. (E) c-MYC is a crucial oncogenic transcription factor mediating gene transcription that is involved in multiple cellular mechanisms, including enhanced cell proliferation and reduced apoptosis. SPAG5 facilitates these processes by interacting with cMYC binding protein (MYCBP). SPAG5 also confers resistance to olaparib by either upregulating the DNA repair proteins RAD51, BRCA1 and BRCA2 or shortening the S-phase duration in which the olaparib-induced DNA lesions occur. (F) MicroRNAs (miRNAs) are small, highly conserved non-coding RNA molecules, some of which may repress cancer progression via SPAG5 in a given cancer type. Figures were created with BioRender.com. 